\title{
Study on the performance of resistance to freeze-thaw of concrete coated with organosilicon hydrophobic agents
}

\author{
SUN Hongyao ${ }^{1, a}$, WANG Xuechuan ${ }^{1,2, b}$, ZHANG Xingduo ${ }^{1, c}$ and SUN Gaoxia ${ }^{1, d}$ \\ ${ }^{1}$ State Key Laboratory of Hydrology-Water Resources and Hydraulic Engineering, Nanjing Hydraulic \\ Research Institute, Nanjing 210029, China \\ 2Jiangsu China Railway ARIT new materials co., LTD, Nanjing 211505, China

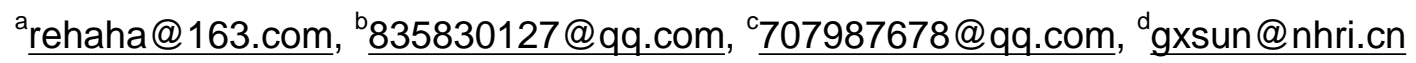

Key words: concrete, silane, freezing and thawing, organosilicon hydrophobic agent, epoxy resin coating, anticorrosion

\begin{abstract}
It is one of the anticorrosion measures of reinforced concrete that the concrete surface coated with organosilicon hydrophobic agents such as silane. In this paper two kinds of water cement ratio (W/C) 0.4 and 0.6 of concrete were selected, silane (isooctyl triethoxysilane) and epoxy resin coating were adopted, and the fresh water and $3.5 \% \mathrm{NaCl}$ solution were set as mediums for freezing and thawing test. The cycle number of freezing and thawing test of concrete treated with silane is 23 more than that of untreated concrete in $0.4 \mathrm{~W} / \mathrm{C}$, and that is 25 more in $0.6 \mathrm{~W} / \mathrm{C}$. The number of freezing and thawing test cycles of concrete treated with silane in fresh water is 47 more cycles than that in $\mathrm{NaCl}$ solution in $0.4 \mathrm{~W} / \mathrm{C}$, and that is 40 more cycles in $0.6 \mathrm{~W} / \mathrm{C}$. The larger the water cement ratio, the worse the performance of resistance to freezing and thawing of concrete is. The damage degree of concrete in $\mathrm{NaCl}$ solution is more serious than that in fresh water.The results show that the performance of resistance to freeze-thaw of treated concrete with epoxy coating is not improved. The performance of resistance to freezing and thawing of concrete can be significantly improved by coating silane on the concrete at the same water cement ratio, but the damage cannot be prevent finally during freezing and thawing. The damage mechanism of treated concrete was discussed during freeze-thaw process.
\end{abstract}

\section{Introduction}

The performance of resistance to freeze-thaw of concrete in cold climate is an important durability index. If the concrete is not protected in the extremely cold environment, the damage of concrete caused by freeze-thaw may be rather serious. The moisture in concrete affects the performance of resistance to freeze-thaw of concrete directly [1].

A hydrophobic layer shall be formed on the concrete surface and in the pore when the organosilicon hydrophobic agents are coated on the concrete, which does not block or seal the concrete pore and keeps the property of gas permeable of concrete. In the alkaline condition of concrete, the alkoxy group in the molecular of organosilicon hydrophobic agents (e.g., alkylalkoxysilane) shall hydrolyze and condensate with inorganic silicate of concrete and the alkyl group arranging on the concrete surface can repel water for protecting concrete [2].

The test results from Frentzel [3] indicated that the weight loss and the decrease of relative dynamic elasticity modulus of concrete were delayed at the early 28 freeze-thaw test cycles while the concrete was treated with silane. The surface spalling phenomenon became serious and the concrete began to deteriorate after 28 cycles. The weight loss of treated concrete was almost the same as that of untreated concrete after 40 cycles. The rapid freeze-thaw test of concrete treated with silane with water cement ratio (W/C) 0.52 was done by Zhao Shangchuan [4]. The results showed that the performance of resistance to freeze-thaw of concrete after treated with silane was greatly improved. The compactness of concrete affected the performance of resistance to freeze-thaw of treated concrete. The cycle number of resistant to freeze-thaw of concrete treated with silane is at least 20 more than that of untreated concrete in the specification [5]. The result from Xu Sangzhen [6] also indicated that 
the cycle number of resistance to freeze-thaw of the concrete after treated with silane was increased by $33 \%$. However, the result from reference [7] showed that the performance of resistance to freeze and thaw of concrete treated with silane was not affected in the deicing salt environment.

Zhang Shiping and other [8] think that no expansion pressure of ice during freezing will be generated when the untreated concrete moisture content is less than $91.7 \%$ of pore volume. However, there are few studies on the mechanism of damage of concrete treated with organosilicon hydrophobic agents such as silane during freezing and thawing process and there are still a lot of questions to be answered on the durability of resistance to freezing and thawing of treated concrete.

\section{Materials and Specimen forming}

Materials. Organosilicon hydrophobic agent: isooctyl triethoxy silane (SILRES ${ }^{\circledR}$ BS 1701). Coating: Epoxy resin coating. Cement: P. O 42.5 Portland cement. Fine aggregate: natural sand, fineness modulus of 2.8. Coarse aggregate: gravel, average size of particles 5-20mm. Mixing water: tap water. Specimen forming. Table 1 is the concrete mix ratio and its compressive strength. According to Table 1, 10 groups of concrete specimens with size $100 \mathrm{~mm} \times 100 \mathrm{~mm} \times 400 \mathrm{~mm}$ were formed, of which 5 groups of concrete were for 0.4 water cement ratio and 5 groups for $0.6 \mathrm{~W} / \mathrm{C}$. After $24 \mathrm{~h}$ the concrete specimens were mold unloaded and placed in standard curing room for $28 \mathrm{~d}$. Then they were taken out and placed in the dry-shrinkage lab for $60 \mathrm{~d}$ in order to reduce the influence of hydration. The details of treating concrete and the conditions of the freeze-thaw test are listed in Table 2 . All faces of concrete were coated. All specimens then were placed in the dry-shrinkage lab for 14d.

Table 1 . The concrete mix ratio and its compressive strength

\begin{tabular}{|c|c|c|c|c|c|c|}
\hline \multirow{2}{*}{$\mathrm{W} / \mathrm{C}$} & \multicolumn{4}{|c|}{ Weight of material per $\mathrm{m}^{3} / \mathrm{kg}$} & \multirow{2}{*}{$\begin{array}{c}\text { sand coarse } \\
\text { aggregate ratio }\end{array}$} & \multirow{2}{*}{$\begin{array}{c}\text { compressive } \\
\text { strength/ MPa }\end{array}$} \\
\hline & water & cement & sand & gravel & & \\
\hline 0.4 & 175 & 437 & 672 & 1096 & 0.38 & 47.3 \\
\hline 0.6 & 195 & 325 & 781 & 1079 & 0.42 & 32.6 \\
\hline
\end{tabular}

Table 2. Details of the freeze-thaw cycle test

\begin{tabular}{cccc}
\hline Sample mark & Treated methods & water cement ratio & freeze-thaw medium \\
\hline FT-KH4 & Not treated & 0.4 & water \\
FT-KH6 & Not treated & 0.6 & water \\
FT-TH4 & Epoxy resin coating & 0.4 & water \\
FT-TH6 & Epoxy resin coating & 0.6 & water \\
FT-SH4 & silane & 0.4 & water \\
FT-SH6 & silane & 0.6 & water \\
FT-KC4 & Not treated & 0.4 & $3.5 \% \mathrm{NaCl}$ solution \\
FT-KC6 & Not treated & 0.6 & $3.5 \% \mathrm{NaCl}$ solution \\
FT-SC4 & silane & 0.4 & $3.5 \% \mathrm{NaCl}$ solution \\
FT-SC6 & silane & 0.6 & $3.5 \% \mathrm{NaCl}$ solution \\
\hline
\end{tabular}

\section{Test methods}

The determination of penetrating depth of silane. The specimens treated with silane were taken out from the dry-shrinkage lab and parallel lines with $10 \mathrm{~mm}$ spacing were drawn on the test face. Then they were split by the compression-testing machine with the test face upper and perpendicular to the direction of parallel line. The methylene blue water solution was sprayed on the fracture surface and the non discoloration area was the permeation area of silane. Each penetration depth can be obtained by gauge according to each parallel line, and the mean value of 9 penetration depths is used as the penetrating depth of silane in each specimen.

Water absorption increment test before freeze-thaw test. The specimens were dried at $(50 \pm 3)^{\circ} \mathrm{C}$ in the oven for $24 \mathrm{~h}$ and then they were taken out to be cooled to $(23 \pm 2)^{\circ} \mathrm{C}$ and weighed. According to Table 2, specimens were put on the glass rods in the flat container which contained water or $3.5 \%$ 
$\mathrm{NaCl}$ solution. The liquid level must be $20 \mathrm{~mm}$ higher than the top surface of concrete. They shall be taken out from the container after $14 \mathrm{~d}$ and weighted after the remaining water on the concrete surface is wiped. The water increment shall be calculated.

Test of resistance to freeze-thaw of concrete treated with silane. It is necessary to determine the water absorption of concrete before the freezing and thawing test. The specimens were immersed in the water or $3.5 \% \mathrm{NaCl}$ solution with the liquid level $20 \mathrm{~mm}$ higher than the top surface of concrete for $14 \mathrm{~d}$ and then they were put into the freezing thawing test box to start test.

The program of freeze-thaw test is set up as below: in each freeze-thaw test cycle, the time is set to $4 \mathrm{~h}$, the minimum temperature is $(-23 \pm 2)^{\circ} \mathrm{C}$, the maximum temperature is $(20 \pm 3)^{\circ} \mathrm{C}$, the temperature in center of specimen is in the range of $7^{\circ} \mathrm{C} \sim-20^{\circ} \mathrm{C}$, the heating time is $90 \mathrm{~min}$ and the cooling time is $150 \mathrm{~min}$ with no clear gap during the process of heating and cooling. In addition, the cooling time from $3{ }^{\circ} \mathrm{C}$ to $-16^{\circ} \mathrm{C}$ is not less than half of the total cooling time. The difference between the center temperature and the surface temperature of the specimen is less than $28^{\circ} \mathrm{C}$.

Every 15 freeze-thaw cycles are taken as a test point where specimens shall be taken out and these datum including concrete surface state and weight loss and the relative dynamic elastic modulus shall be collected. Then the specimens must be placed into the test box upside down and the freeze-thaw test shall be done continually.

Test of weight loss rate. The specimens were taken out from the container after immersed in the medium for $14 \mathrm{~d}$, then they were dried and weighted before the freeze-thaw test. After the freeze-thaw test was started, the specimens were taken out and the residue on the concrete surface was washed away in every 15 freeze-thaw cycles. And each specimen was weighed immediately. The rate of weight loss (hereafter called WL) of each specimen after freeze-thaw test can be calculated with Eq. $W_{n}=\left(G_{n}-G_{0}\right) / G_{0} \times 100 \%$, where $W_{n}$ - the rate of weight loss of specimen after freeze-thaw test, $\%$; $G_{0}$ - the weight of the specimen after immersed in medium for $14 \mathrm{~d}$ before the freeze-thaw test, $\mathrm{g}$; $G_{n}$ - The weight of the specimen after $n$ freeze-thaw test cycles, $\mathrm{g}$.

Test of relative dynamic elastic modulus of concrete. The relative dynamic elastic modulus test equipment must be preheated before test. The relative dynamic elastic modulus (hereafter called RDEM) shall be measured at the beginning of test and the test point of every 15 freeze-thaw cycles. The specimen was placed on the sponge pad with $2 \mathrm{~cm}$ thickness and the transverse natural vibration frequency was obtained. The RDEM can be calculated with Eq. $P_{n}=f_{n}^{2} / f_{0}^{2} \times 100 \%$, where $P_{n}$ - the RDEM of specimen after $n$ freeze-thaw test cycles, $\% ; f_{0}$ - the natural vibration frequency of specimen before freeze-thaw test, $\mathrm{Hz} ; f_{n}$ - the natural vibration frequency of specimen after $n$ freeze-thaw cycles, Hz.

\section{Test results and discussion}

Weight increment of specimen after immersed in the medium. The weight increments of concrete immersed in mediums for $14 \mathrm{~d}$ before freezing and thawing test are shown in Fig. 1. The results indicate that the influence of mediums (water and $\mathrm{NaCl}$ solution) on the weight increment of concrete is slight, but the influence of concrete water cement ratio on it is obvious. The weight increment of concrete treated with silane and epoxy resin coating is lower than that of untreated concrete and that of concrete treated with silane is lower than that treated with epoxy resin coating. The difference of concrete water cement ratio causes difference of the pore structure of concrete. Epoxy coating is a film-forming coating and it seals the concrete pore.

Variation of weight loss and RDEM of concrete during freeze-thaw test. The variation of weight loss and RDEM of concrete are used to evaluate the property of resistance to freeze-thaw of concrete.

Weight loss of concrete during freeze-thaw test. The weight variation curve of concrete immersed in water during freeze-thaw test is shown in Fig. 2a. The results show that the weight of concrete treated with epoxy resin coating and untreated get slight increase at the beginning of freeze-thaw test and then the weights decrease with the test time increasing. Weights of concrete treated with silane increased at first and then decreased. 


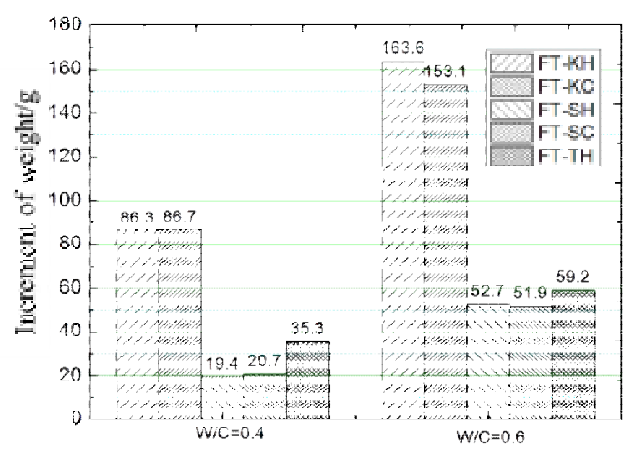

Fig.1. The increment of concrete weight after immersed in mediums for $14 \mathrm{~d}$

The weight of FT-SH6 concrete increased at first 45 freeze-thaw test cycles and showed no weight loss at first 66 freeze-thaw test cycles. The weight of FT-SH4 concrete increased at first 60 test cycles and showed no weight loss at first 92 test cycles. The property of resistance to freeze-thaw of concrete treated with epoxy resin coating is not improved. The start time of weight loss of concrete treated with silane was retarded. The concrete begins to show rapid weight loss after reaching the peak of water absorption.
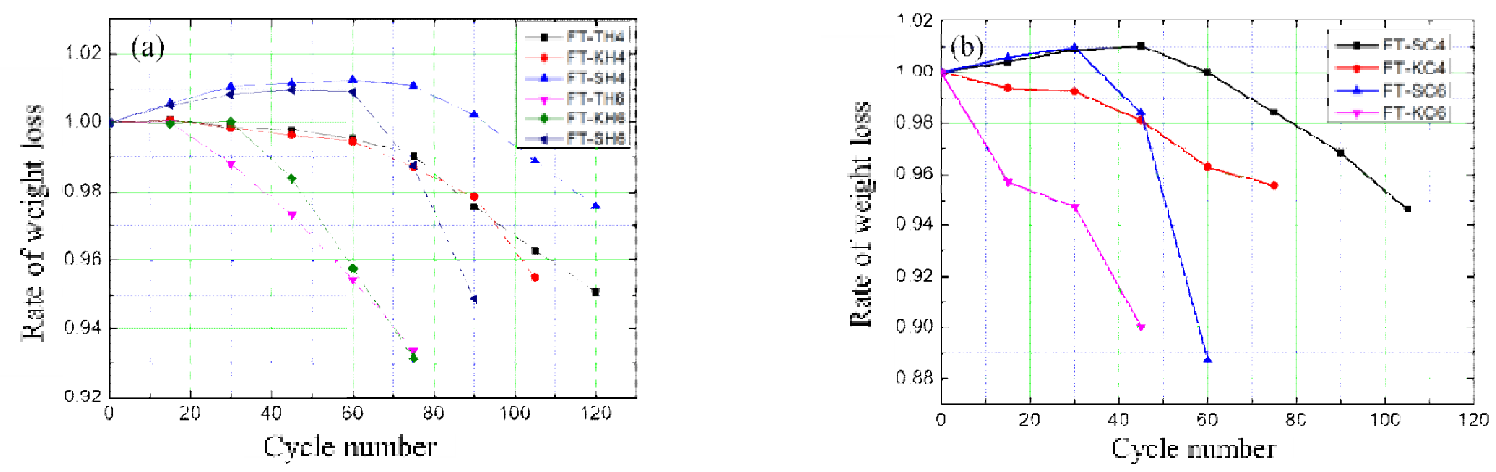

Fig.2. Variation curve of concrete weight during freeze-thaw test (a) in water, (b) in $\mathrm{NaCl}$ solution

The variation curve of concrete weight during freeze-thaw test in $3.5 \% \mathrm{NaCl}$ solution is shown in Fig.2b. From the start time of test, the surface erosion was shown on the untreated concrete surface and the weight of concrete began to decrease. The weight of concrete treated with silane is increasing at first and then decreasing. The weight of FT-SH6 concrete is increasing at first 30 freeze-thaw test cycles and then begins to decrease rapidly. The weight of FT-SH4 concrete is increasing at first 45 freeze-thaw test cycles and then also begins to decrease rapidly. Compared with the results in Fig.2a, the weight loss of concrete in $\mathrm{NaCl}$ solution is more serious than that in water.

Variation of RDEM of concrete during freeze-thaw test. The RDEM of concrete reflects the deterioration process of the concrete. Especially it can reflect the interior damage degree of concrete.
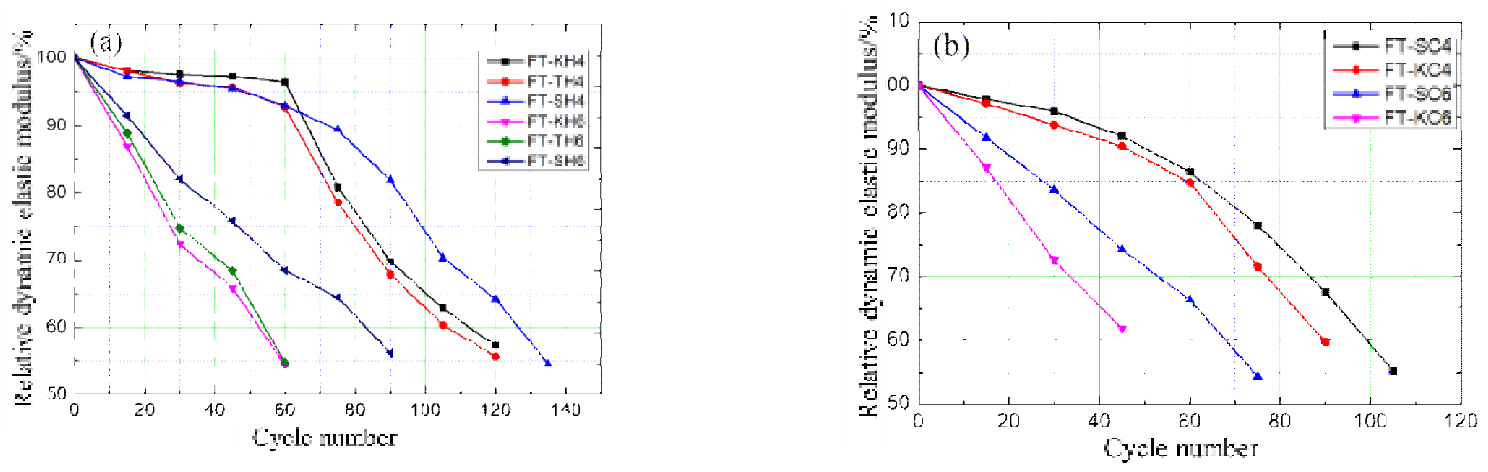

Fig.3. Variation curve of RDEM of concrete during freeze-thaw test (a) in water, (b) in $\mathrm{NaCl}$ solution

The variation curve of RDEM of concrete during freeze-thaw test in water is shown in Fig. 3a. The variation trend of RDEM of concrete treated with epoxy resin coating and untreated is almost similar. 
The property of resistant to freeze-thaw of concrete treated with silane is the best. The property with W/C 0.4 is better than that with W/C 0.6 for the same treated method.

The variation curve of RDEM of concrete during freeze-thaw test in $\mathrm{NaCl}$ solution is shown in Fig. $3 \mathrm{~b}$. When the $\mathrm{W} / \mathrm{C}$ is 0.4 , the concrete treated with silane begins to show better property of resistant to freeze-thaw than untreated concrete after 60 test cycles. When the W/C is 0.6 , it shows better property from the beginning of freeze-thaw test.

Analysis of the results. During the freeze-thaw test, the surface layer of FT-KH4 specimen was completely destroyed including sand dropped largely and coarse aggregate exposed and shedding after 60 test cycles. The hardened cement paste of the surface mortar layer no longer had bonding capacity. The epoxy coating on the FT-TH4 specimen had been completely destroyed and lost the protective effect after 15 test cycles. The subsequent failure process of FT-TH4 specimen is similar to that of the FT-KH4 specimen.

The failure mode of FT-SH4 concrete is different from that of FT-KH4. After 120 test cycles, the surface of the FT-SH4 specimen was damaged. When the failure of concrete occurs, the surface mortar is exfoliated, that is, the hardened cement paste of the surface mortar layer still has some bonding capacity. The reason may be that the hydrophobic layer is formed on the concrete surface and little water is in the pore of hydrophobic layer.

When the weight loss rate reaches $5 \%$ or the relative dynamic elastic modulus decreases to $60 \%$, the corresponding cycle number is the grade of resistance to freeze-thaw of the concrete. The cycle numbers of resistance to freeze-thaw test of concrete in Fig.2 and Fig.3 are summed in Table 3。

Table 3 . The cycle number of resistance to freeze-thaw test of concrete

\begin{tabular}{cccccccc}
\hline \multirow{2}{*}{ W/C } & Treated & \multicolumn{2}{c}{ Water(W) } & \multicolumn{2}{c}{ NaCl solution(N) } & \multicolumn{2}{c}{$\Delta \mathrm{f}^{1}$} \\
& method & WL/5\% & RDEM/60\% & WL5/\% & RDEM/60\% & WL/5\% & RDEM/60\% \\
\hline \multirow{4}{*}{0.4} & Untreated(K) & 109 & 113 & 80 & 90 & 29 & 23 \\
& Coating(T) & 120 & 105 & n/a & n/a & n/a & n/a \\
& Silane(S) & 150 & 126 & 103 & 100 & 47 & 26 \\
& $\Delta \mathrm{t}^{2}$ & 41 & 13 & 23 & 10 & 18 & 3 \\
& Untreated(K) & 65 & 53 & 25 & 46 & 40 & 7 \\
& Coating(T) & 64 & 55 & n/a & n/a & n/a & n/a \\
& Silane(S) & 90 & 82 & 50 & 68 & 40 & 14 \\
& $\Delta \mathrm{t}^{2}$ & 25 & 29 & 25 & 22 & 0 & 7 \\
\hline
\end{tabular}

Note: $1 \Delta \mathrm{f}=(\mathrm{W}-\mathrm{N}) ; 2 \Delta \mathrm{t}=(\mathrm{S}-\mathrm{K})$

The results in Table 3 show that the damage of specimens in $\mathrm{NaCl}$ solution is more serious than that in water and that with bigger $\mathrm{W} / \mathrm{C}$ is more serious. The property of resistant to freeze-thaw of concrete treated with silane is improved. Compared the cycle number of the WL with that of the RDEM at the same treat method, the corresponding cycles of freeze-thaw test are different and some differences exceed 20 test cycles. So the same evaluation index must be adopted when comparing the property of resistance to freeze-thaw of concrete treated with different methods. When the W/C is 0.6 , the $\Delta \mathrm{t}$ is almost the same between WL and RDEM, so the concrete with $0.6 \mathrm{~W} / \mathrm{C}$ can be used to determine the property of resistance to freeze-thaw of concrete treated with silane.

The property of resistance to freeze-thaw of concrete treated with epoxy resin coating is not improved. The reason may be that the linear expansion coefficient between concrete and epoxy coating is different and the epoxy coating on the concrete surface shall be fracture under repeated high and low temperature. When the concrete is treated with silane, the hydrophobic layer is formed on the concrete surface and the gas permeability is kept. Because there is no failure caused by the difference of linear expansion coefficient and the rate of water absorption of concrete treated with silane is less than that of untreated concrete, the property of resistance to freeze-thaw of concrete treated with silane is improved obviously.

In conclusion, the start time of failure of treated concrete with silane caused by freezing and thawing can be delayed but the failure cannot be stopped finally. 
Discussion on the mechanism of resistance to freeze-thaw of concrete treated with silane. If there are no defects caused by abnormal factors such as cracks in concrete, the concrete pore is the effective transportation path of medium during freeze-thaw process.

There will be pressure difference $P_{\mathrm{s}}$ on both sides of the concave liquid surface which is formed in the concrete pore. If the concave liquid surface is looked as the spherical surface, according to the Laplace equation, the pressure difference $P_{\mathrm{s}}$ can be expressed by the formula: $P_{\mathrm{s}}=2 \gamma \cos \theta / \mathrm{r}$, where $P_{\mathrm{s}}$ - pressure, $\mathrm{Pa} ; \gamma$ - liquid surface tension coefficient, $\mathrm{N} / \mathrm{m} ; r$ - pore radius, $\mathrm{m} ; \theta$ - contact angle of water in concrete pore.

After the concrete is coated with silane, the hydrophobic layer with molecular thickness is formed, which does not change the pore distribution and pore characteristics of concrete. However, the surface tension decreases and the water contact angle on the concrete surface is greater than $90^{\circ}$, so the pressure difference $P_{\mathrm{s}}$ is formed with the outward force direction in the pores.

When the water seals the concrete pore opening, the gas in the pore can be considered as the gas in a sealed container. The pressure $P_{\mathrm{t}}$ shall change with the variation of temperature, and the pressure $P_{\mathrm{t}}$ meets the gas state equation: $P_{\mathrm{t}}=k\left(T_{1}-T_{2}\right) / T_{0}$, where $P_{\mathrm{t}}$-pressure caused by variation of temperature, $\mathrm{Pa}$; $k$-constant for pressure conversion, $1.01 \times 10^{5} \mathrm{~Pa} ; T_{1}$-temperature before variation, $\mathrm{K} ; T_{2}-$ temperature after variation, $\mathrm{K} ; T_{0}$ - freezing temperature, $273.15 \mathrm{~K}$ in water.

The equation of the surface tension coefficient $\gamma$ at temperature $T$ was given by the reference [9] ( $\left.\mathrm{a}_{10}\right)\left(\gamma=94.63 \times 10^{-3}+2.18 \times 10^{-6} T-2.63 \times 10^{-7} T^{2}\right)$. After the concrete is coated with silane, the pressure difference $\Delta P$ in concrete pore can be expressed with the equation: $\Delta P=P_{\mathrm{s}}-P_{\mathrm{t}}=2 \gamma \cos \theta / r-k\left(T_{1}-T_{2}\right) / T_{0}$. If the surface contact angle $\theta$ is set to $125^{\circ}, T_{1}$ is set to $293.15 \mathrm{~K}$ and $T_{0}$ is set to $273.15 \mathrm{~K}$, the relation between pressure difference $\Delta P$ and the pore radius $r$ at $T_{2}$ is an inverse function relation.

If the pore radius $r$ is set as a constant, the gas contraction pressure $P_{\mathrm{t}}$ in the pore increases with the decrease of temperature $T_{2}$. When the $\Delta P$ is negative, the water on the concrete surface shall be absorbed into the concrete pore. If the ice is formed during freezing, the increasing negative pressure of gas in the pore cannot break the concrete. But if the ice is not converted to water during thawing, the increasing gas expanding pressure $P_{\mathrm{t}}$ in the pore may break the concrete. The bigger the water cement ratio is, the bigger the concrete pore radius is, and the greater the water absorbed is. This is the reason why the property of resistance to freeze-thaw of concrete treated with silane with the W/C of 0.4 is better than that with W/C of 0.6 .

If $T_{2}$ is set to the freezing point temperature, the freezing temperature of $\mathrm{NaCl}$ solution is lower than that of water. The amount of water absorption in $\mathrm{NaCl}$ solution shall be more than that in water during freezing. The concrete surface can be broken by the volume expansion pressure of ice during freezing and the swelling pressure of gas in the pore during thawing. This is the reason why the degree of damage of concrete surface treated with silane in $\mathrm{NaCl}$ solution is more serious than that in water.

Because the surface of untreated concrete pore is hydrophilic surface, water shall fill the pore gradually. The swelling volume of ice formed from water may destroy the pore during freezing, so the weight of concrete begins to lose at the start of freezing. When the pressure difference $\Delta P$ is 0 , different temperatures $T_{2}$ relates to different pore radius $r$ of concrete treated with silane. The result informs that there is a critical pore radius $r$ during freezing. If the pore radius is bigger than the critical pore radius, the water shall be absorbed into concrete. Because the concrete treated with silane cannot be broken by little water which is absorbed into concrete pore at the preliminary freeze-thaw test and the little water is remained in the concrete, so the weight of concrete is increasing. But once the damage of concrete surface occurs, the water shall be absorbed into concrete quickly and the degree of damage shall become more and more serious. So the property of resistance to freeze-thaw of concrete treated with silane is better than that of untreated concrete and the cycle number of resistance to freeze-thaw test of treated concrete is about 20 more cycles than that of untreated concrete. This is the reason why the damage cannot be prevented finally by the method of treating concrete with silane during freeze-thaw process. 


\section{Conclusions}

The performance of resistance to freeze-thaw of concrete treated with epoxy resin coating is not improved. But that of concrete treated with silane is improved. The lower the water cement ratio of concrete is, the better the property of resistance to freeze-thaw of concrete treated with silane is. The degree of damage of concrete treated with silane in $\mathrm{NaCl}$ solution is more serious than that in water. There is difference while using method of weight loss of concrete and method of the decrease of relative dynamic elasticity modulus of concrete to evaluate the performance of resistance to freeze-thaw of treated concrete. But the difference of $\Delta \mathrm{t}$ is so small that the treated concrete with water cement ratio of 0.6 can be used to evaluate the performance of resistance to freeze-thaw test.

There are two forces in concrete pore which are the repulsive force $P_{\mathrm{s}}$ at hydrophobic layer and the gas contraction or swelling force $P_{\mathrm{t}}$. The relation between the pressure difference $\Delta P\left(P_{\mathrm{s}}-P_{\mathrm{t}}\right)$ and the pore radius $r$ is an inverse function relation. The damage of treated concrete may be caused by the volume expansion pressure of ice during freezing and the gas swelling pressure in concrete pore during thawing. The performance of resistance to freeze-thaw of concrete can be improved by the method of coating silane on the concrete, but the damage cannot be prevented finally during freeze-thaw process.

\section{Acknowledgment}

This work is financially supported by the National Natural Science Foundation of the P. R. China (51279110).

\section{References}

[1] LIU Xiaojuan, YAN Jun, et al. Technology of Waterproofing Treatment of Silane-Based Water-Proof Agent and Its Application on Concrete Structure. China Building Waterproof, 2004(10): 6-10..

[2] H.Y. SUN, Z. YANG, G.L. SHAN, et al. Current Situation of Research and Application of Silicone Water Repellent for Protecting Reinforced Concrete. Bridge Maintenance, Safety, Management and Life Extension - Chen, Frangopol \& Ruan (Eds) .Taylor \& Francis Group, London, 978-1-138-00103-9. 2014:2495-2502.

[3] Frentzel,F.A. Finger, Bauhaus. Water Uptake and Scaling of Impregnated Pavement Concrete under Freeze Thaw and De-Icing Agent Attack. 4th International Conference on Water Repellent Treatment of Building Materials Aedificatio Publishers, 223-240 (2005).

[4] ZHAO Shang-chuan, YU Hai. Research on Water Uptake and Freeze-Thaw Stability of Impregnated Concrete by Silane. Concrete, 2008(11):80-82.

[5] JTG/T B07-01-2006. Specification for Deterioration Prevention of Highway Concrete Structures. Beijing: China Communications Press, 2006.

[6] XU Sang-Zhen, WS Chi, QT Liu, KX Lin. Experimental Study on Performance of Airport Pavement Concrete Impregnated with Silane. Concrete, 2012(7):67-70.

[7] Perenchio W F. Durability of Concrete Treated with Silanes. Concrete International, 1988, 10(11):34-40.

[8] ZHANG Shi-ping, DENG Min, TANG Ming-shu. Advance in Research on Damagement of Concrete Due to Freeze-thaw Cycles. Journal of Materials Science \&. Engineering, 2008, (6):990-994. 
[9] HU Fene, WEI Shengxian, SHI Youming, LI Dongyu. A Comparative Study on the Equations Between the Surface Tension Coefficient of Water and the Temperature. Journal of Qujing Normal University, 2015, 34(3):7-10. 